\title{
$q$-analogue of a new sequence of linear positive operators
}

\author{
Vijay Gupta', Taekyun Kim² ${ }^{2 *}$ and Sang-Hun Lee ${ }^{3}$
}

"Correspondence: tkkim@kw.ac.kr ${ }^{2}$ Department of Mathematics, Kwangwoon University, Seoul 139-701, S. Korea

Full list of author information is available at the end of the article

\begin{abstract}
This paper deals with Durrmeyer type generalization of q-Baskakov type operators using the concept of $q$-integral, which introduces a new sequence of positive $q$-integral operators. We show that this sequence is an approximation process in the polynomial weighted space of continuous functions defined on the interval $[0, \infty)$. An estimate for the rate of convergence and weighted approximation properties are also obtained.
\end{abstract}

MSC: Primary 41A25; 41A36

Keywords: Durrmeyer type operators; weighted approximation; rate of convergence; $q$-integral

\section{Introduction}

In the year 2003 Agrawal and Mohammad [1] introduced a new sequence of linear positive operators by modifying the well-known Baskakov operators having weight functions of Szasz basis function as

$$
\mathcal{D}_{n}(f, x)=n \sum_{k=1}^{\infty} p_{n, k}(x) \int_{0}^{\infty} s_{n, k-1}(t) f(t) d t+p_{n, 0}(x) f(0), \quad x \in[0, \infty)
$$

where

$$
p_{n, k}(x)=\left(\begin{array}{c}
n+k-1 \\
k
\end{array}\right) \frac{x^{k}}{(1+x)^{n+k}}, \quad s_{n, k}(t)=e^{-n t} \frac{(n t)^{k}}{k !} .
$$

It is observed in [1] that these operators reproduce constant as well as linear functions. Later, some direct approximation results for the iterative combinations of these operators were studied in [14].

A lot of works on $q$-calculus are available in literature of different branches of mathematics and physics. For systematic study, we refer to the work of Ernst [5], Kim [10, 11], and Kim and Rim [9]. The application of $q$-calculus in approximation theory was initiated by Phillips [13], who was the first to introduce $q$-Bernstein polynomials and study their approximation properties. Very recently the $q$-analogues of the Baskakov operators and their Kantorovich and Durrmeyer variants have been studied in [2,3] and [7] respectively. We recall some notations and concepts of $q$-calculus. All of the results can be found in [5] and [8]. In what follows, $q$ is a real number satisfying $0<q<1$.

C) 2012 Gupta et al.; licensee Springer. This is an Open Access article distributed under the terms of the Creative Commons Attribution License (http://creativecommons.org/licenses/by/2.0), which permits unrestricted use, distribution, and reproduction in any medium, provided the original work is properly cited. 
For $n \in \mathbb{N}$,

$$
\begin{aligned}
{[n]_{q}: } & =\frac{1-q^{n}}{1-q}, \\
{[n]_{q} ! } & := \begin{cases}{[n]_{q}[n-1]_{q} \cdots[1]_{q},} & n=1,2, \ldots, \\
1, & n=0 .\end{cases}
\end{aligned}
$$

The $q$-binomial coefficients are given by

$$
\left[\begin{array}{l}
n \\
k
\end{array}\right]_{q}=\frac{[n]_{q} !}{[k]_{q} ![n-k]_{q} !}, \quad 0 \leq k \leq n
$$

The $q$-Beta integral is defined by [12]

$$
\Gamma_{q}(t)=\int_{0}^{\frac{1}{1-q}} x^{t-1} E_{q}(-q x) d_{q} x, \quad t>0
$$

which satisfies the following functional equation:

$$
\Gamma_{q}(t+1)=[t]_{q} \Gamma_{q}(t), \quad \Gamma_{q}(1)=1
$$

For $f \in C[0, \infty), q>0$ and each positive integer $n$, the $q$-Baskakov operators [2] are defined as

$$
\begin{aligned}
\mathcal{B}_{n, q}(f, x) & =\sum_{k=0}^{\infty}\left[\begin{array}{c}
n+k-1 \\
k
\end{array}\right]_{q} q^{\frac{k(k-1)}{2}} \frac{x^{k}}{(1+x)_{q}^{n+k}} f\left(\frac{[k]_{q}}{q^{k-1}[n]_{q}}\right) \\
& =\sum_{k=0}^{\infty} p_{n, k}^{q}(x) f\left(\frac{[k]_{q}}{q^{k-1}[n]_{q}}\right)
\end{aligned}
$$

where

$$
(1+x)_{q}^{n}:= \begin{cases}(1+x)(1+q x) \cdots\left(1+q^{n-1} x\right), & n=1,2, \ldots \\ 1, & n=0 .\end{cases}
$$

Remark 1 The first three moments of the $q$-Baskakov operators are given by

$$
\begin{aligned}
& \mathcal{B}_{n, q}(1, x)=1, \\
& \mathcal{B}_{n, q}(t, x)=x, \\
& \mathcal{B}_{n, q}\left(t^{2}, x\right)=x^{2}+\frac{x}{[n]_{q}}\left(1+\frac{1}{q} x\right) .
\end{aligned}
$$

As the operators $\mathcal{D}_{n}(f, x)$ have mixed basis functions in summation and integration and have an interesting property of reproducing linear functions, we were motivated to study these operators further. Here we define the $q$-analogue of the operators as

$$
\mathcal{D}_{n}^{q}(f, x)=[n]_{q} \sum_{k=1}^{\infty} p_{n, k}^{q}(x) \int_{0}^{q /\left(1-q^{n}\right)} q^{-k} s_{n, k-1}^{q}(t) f\left(t q^{-k}\right) d_{q} t+p_{n, 0}^{q}(x) f(0)
$$


where $x \in[0, \infty)$ and

$$
p_{n, k}^{q}(x)=\left[\begin{array}{c}
n+k-1 \\
k
\end{array}\right]_{q} q^{\frac{k(k-1)}{2}} \frac{x^{k}}{(1+x)_{q}^{n+k}}, \quad s_{n, k}^{q}(t)=E_{q}\left(-[n]_{q} t\right) \frac{\left([n]_{q} t\right)^{k}}{[k]_{q} !} .
$$

In case $q=1$, the above operators reduce to the operators (1.1). In the present paper, we estimate a local approximation theorem and the rate of convergence of these new operators as well as their weighted approximation properties.

\section{Moment estimation}

Lemma 1 The following equalities hold:

(i) $\mathcal{D}_{n}^{q}(1, x)=1$,

(ii) $\mathcal{D}_{n}^{q}(t, x)=x$,

(iii) $\mathcal{D}_{n}^{q}\left(t^{2}, x\right)=x^{2}+\frac{x}{[n]_{q}}\left(1+q+\frac{x}{q}\right)$.

Proof The operators $\mathcal{D}_{n}^{q}$ are well defined on the function $1, t, t^{2}$. Then for every $x \in[0, \infty)$, we obtain

$$
\mathcal{D}_{n}^{q}(1, x)=[n]_{q} \sum_{k=1}^{\infty} p_{n, k}^{q}(x) \int_{0}^{q /\left(1-q^{n}\right)} q^{-k} \frac{\left([n]_{q} t\right)^{k-1}}{[k-1]_{q} !} E_{q}\left(-[n]_{q} t\right) d_{q} t+p_{n, 0}^{q}(x) .
$$

Substituting $[n]_{q} t=q y$ and using (1.2), we have

$$
\begin{aligned}
\mathcal{D}_{n}^{q}(1, x) & =[n]_{q} \sum_{k=1}^{\infty} p_{n, k}^{q}(x) \int_{0}^{1 /(1-q)} q^{-k} \frac{(q y)^{k-1}}{[k-1]_{q} !} E_{q}(-q y) \frac{q d_{q} y}{[n]_{q}}+p_{n, 0}^{q}(x) \\
& =\sum_{k=1}^{\infty} p_{n, k}^{q}(x)+p_{n, 0}^{q}(x)=\mathcal{B}_{n, q}(1, x)=1,
\end{aligned}
$$

where $\mathcal{B}_{n, q}(f, x)$ is the $q$-Baskakov operator defined by (1.3).

Next, we have

$$
\mathcal{D}_{n}^{q}(t, x)=[n]_{q} \sum_{k=1}^{\infty} p_{n, k}^{q}(x) \int_{0}^{q /\left(1-q^{n}\right)} q^{-k} \frac{\left([n]_{q} t\right)^{k-1}}{[k-1]_{q} !} E_{q}\left(-[n]_{q} t\right) t q^{-k} d_{q} t .
$$

Again substituting $[n]_{q} t=q y$ and using (1.2), we have

$$
\begin{aligned}
\mathcal{D}_{n}^{q}(t, x) & =[n]_{q} \sum_{k=1}^{\infty} p_{n, k}^{q}(x) \int_{0}^{1 /(1-q)} q^{-k} \frac{(q y)^{k}}{[k-1]_{q} ![n]_{q}} E_{q}(-q y) \frac{q d_{q} y}{[n]_{q} q^{k}} \\
& =\sum_{k=0}^{\infty} p_{n, k}^{q}(x) q \frac{[k]_{q}}{[n]_{q} q^{k}}=\mathcal{B}_{n, q}(t, x)=x .
\end{aligned}
$$

Finally,

$$
\mathcal{D}_{n}^{q}\left(t^{2}, x\right)=[n]_{q} \sum_{k=0}^{\infty} p_{n, k}^{q}(x) \int_{0}^{q /\left(1-q^{n}\right)} q^{-k} \frac{\left([n]_{q} t\right)^{k-1}}{[k-1]_{q} !} E_{q}\left(-[n]_{q} t\right) t^{2} q^{-2 k} d_{q} t .
$$


Again substituting $[n]_{q} t=q y$, using $(1.2)$ and $[k+1]_{q}=[k]_{q}+q^{k}$, we have

$$
\begin{aligned}
\mathcal{D}_{n}^{q}\left(t^{2}, x\right) & =[n]_{q} \sum_{k=1}^{\infty} p_{n, k}^{q}(x) \int_{0}^{1 /(1-q)} q^{-k} \frac{(q y)^{k+1}}{[k-1]_{q} ![n]_{q}^{2}} E_{q}(-q y) q^{-2 k} \frac{q d_{q} y}{[n]_{q}} \\
& =\sum_{k=1}^{\infty} p_{n, k}^{q}(x) \frac{[k+1]_{q}[k]_{q}}{[n]_{q}^{2} q^{2 k-2}} \\
& =\sum_{k=1}^{\infty} p_{n, k}^{q}(x) \frac{\left([k]_{q}+q^{k}\right)[k]_{q}}{[n]_{q}^{2} q^{2 k-2}} \\
& =\mathcal{B}_{n, q}\left(t^{2}, x\right)+\frac{q}{[n]_{q}} \mathcal{B}_{n, q}(t, x)=x^{2}+\frac{x}{[n]_{q}}\left(1+q+\frac{x}{q}\right) .
\end{aligned}
$$

Remark 2 If we put $q=1$, we get the moments of a new sequence $\mathcal{D}_{n}(f, x)$ considered in [1] as operators as

$$
\begin{aligned}
& \mathcal{D}_{n}(t-x, x)=0, \\
& \mathcal{D}_{n}\left((t-x)^{2}, x\right)=\frac{x(x+2)}{n} .
\end{aligned}
$$

Lemma 2 Let $q \in(0,1)$, then for $x \in[0, \infty)$ we have

$$
\mathcal{D}_{n}^{q}\left((t-x)^{2}, x\right)=\frac{x\left(x+q[2]_{q}\right)}{q[n]_{q}} .
$$

\section{Direct theorems}

By $C_{B}[0, \infty)$ we denote the space of real valued continuous bounded functions $f$ on the interval $[0, \infty)$; the norm- $\|\cdot\|$ on the space $C_{B}[0, \infty)$ is given by

$$
\|f\|=\sup _{0 \leq x<\infty}|f(x)| .
$$

The Peetre's $K$-functional is defined by

$$
K_{2}(f, \delta)=\inf \left\{\|f-g\|+\delta\left\|g^{\prime \prime}\right\|: g \in W_{\infty}^{2}\right\}
$$

where $W_{\infty}^{2}=\left\{g \in C_{B}[0, \infty): g^{\prime}, g^{\prime \prime} \in C_{B}[0, \infty)\right\}$. By [4, pp.177], there exists a positive constant $C>0$ such that $K_{2}(f, \delta) \leq C \omega_{2}\left(f, \delta^{1 / 2}\right), \delta>0$ and the second order modulus of smoothness is given by

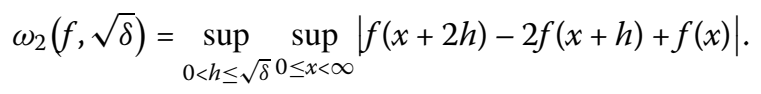

Also, for $f \in C_{B}[0, \infty)$ a usual modulus of continuity is given by

$$
\omega(f, \delta)=\sup _{0<h \leq \delta} \sup _{0 \leq x<\infty}|f(x+h)-f(x)| .
$$


Theorem 1 Let $f \in C_{B}[0, \infty)$ and $0<q<1$. Then for all $x \in[0, \infty)$ and $n \in N$, there exists an absolute constant $C>0$ such that

$$
\left|\mathcal{D}_{n}^{q}(f, x)-f(x)\right| \leq C \omega_{2}\left(f, \sqrt{\frac{x\left(x+q[2]_{q}\right)}{q[n]_{q}}}\right) .
$$

Proof Let $g \in W_{\infty}^{2}$ and $x, t \in[0, \infty)$. By Taylor's expansion, we have

$$
g(t)=g(x)+g^{\prime}(x)(t-x)+\int_{x}^{t}(t-u) g^{\prime \prime}(u) d u
$$

Applying Lemma 2, we obtain

$$
\mathcal{D}_{n}^{q}(g, x)-g(x)=\mathcal{D}_{n}^{q}\left(\int_{x}^{t}(t-u) g^{\prime \prime}(u) d u, x\right) .
$$

Obviously, we have $\left|\int_{x}^{t}(t-u) g^{\prime \prime}(u) d u\right| \leq(t-x)^{2}\left\|g^{\prime \prime}\right\|$. Therefore,

$$
\left|\mathcal{D}_{n}^{q}(g, x)-g(x)\right| \leq \mathcal{D}_{n}^{q}\left((t-x)^{2}, x\right)\left\|g^{\prime \prime}\right\|=\frac{x\left(x+q[2]_{q}\right)}{q[n]_{q}}\left\|g^{\prime \prime}\right\| .
$$

Using Lemma 1, we have

$$
\left|\mathcal{D}_{n}^{q}(f, x)\right| \leq[n]_{q} \sum_{k=1}^{\infty} p_{n, k}^{q}(x) \int_{0}^{q /\left(1-q^{n}\right)} q^{-k} s_{n, k-1}^{q}(t)\left|f\left(t q^{-k}\right)\right| d_{q} t+p_{n, 0}^{q}(x)|f(0)| \leq\|f\| .
$$

Thus

$$
\begin{aligned}
\left|\mathcal{D}_{n}^{q}(f, x)-f(x)\right| & \leq\left|\mathcal{D}_{n}^{q}(f-g, x)-(f-g)(x)\right|+\left|\mathcal{D}_{n}^{q}(g, x)-g(x)\right| \\
& \leq 2\|f-g\|+\frac{x\left(x+q[2]_{q}\right)}{q[n]_{q}}\left\|g^{\prime \prime}\right\| .
\end{aligned}
$$

Finally, taking the infimum over all $g \in W_{\infty}^{2}$ and using the inequality $K_{2}(f, \delta) \leq C \omega_{2}\left(f, \delta^{1 / 2}\right)$, $\delta>0$, we get the required result. This completes the proof of Theorem 1 .

We consider the following class of functions:

Let $H_{x^{2}}[0, \infty)$ be the set of all functions $f$ defined on $[0, \infty)$ satisfying the condition $|f(x)| \leq M_{f}\left(1+x^{2}\right)$, where $M_{f}$ is a constant depending only on $f$. By $C_{x^{2}}[0, \infty)$, we denote the subspace of all continuous functions belonging to $H_{x^{2}}[0, \infty)$. Also, let $C_{x^{2}}^{*}[0, \infty)$ be the subspace of all functions $f \in C_{x^{2}}[0, \infty)$, for which $\lim _{|x| \rightarrow \infty} \frac{f(x)}{1+x^{2}}$ is finite. The norm on $C_{x^{2}}^{*}[0, \infty)$ is $\|f\|_{x^{2}}=\sup _{x \in[0, \infty)} \frac{|f(x)|}{1+x^{2}}$. We denote the modulus of continuity of $f$ on closed interval $[0, a], a>0$ as by

$$
\omega_{a}(f, \delta)=\sup _{|t-x| \leq \delta} \sup _{x, t \in[0, a]}|f(t)-f(x)| .
$$

We observe that for function $f \in C_{x^{2}}[0, \infty)$, the modulus of continuity $\omega_{a}(f, \delta)$ tends to zero. 
Theorem 2 Let $f \in C_{x^{2}}[0, \infty), q \in(0,1)$ and $\omega_{a+1}(f, \delta)$ be its modulus of continuity on the finite interval $[0, a+1] \subset[0, \infty)$, where $a>0$. Then for every $n>2$,

$$
\left\|\mathcal{D}_{n}^{q}(f)-f\right\|_{C[0, a]} \leq \frac{6 M_{f} a\left(1+a^{2}\right)(2+a)}{q[n]_{q}}+2 \omega\left(f, \sqrt{\frac{a\left(a+q[2]_{q}\right)}{q[n]_{q}}}\right) .
$$

Proof For $x \in[0, a]$ and $t>a+1$, since $t-x>1$, we have

$$
\begin{aligned}
|f(t)-f(x)| & \leq M_{f}\left(2+x^{2}+t^{2}\right) \\
& \leq M_{f}\left(2+3 x^{2}+2(t-x)^{2}\right) \\
& \leq 6 M_{f}\left(1+a^{2}\right)(t-x)^{2} .
\end{aligned}
$$

For $x \in[0, a]$ and $t \leq a+1$, we have

$$
|f(t)-f(x)| \leq \omega_{a+1}(f,|t-x|) \leq\left(1+\frac{|t-x|}{\delta}\right) \omega_{a+1}(f, \delta)
$$

with $\delta>0$.

From (3.1) and (3.2) we can write

$$
|f(t)-f(x)| \leq 6 M_{f}\left(1+a^{2}\right)(t-x)^{2}+\left(1+\frac{|t-x|}{\delta}\right) \omega_{a+1}(f, \delta)
$$

for $x \in[0, a]$ and $t \geq 0$. Thus

$$
\begin{aligned}
\left|\mathcal{D}_{n}^{q}(f, x)-f(x)\right| \leq & \mathcal{D}_{n}^{q}(|f(t)-f(x)|, x) \\
\leq & 6 M_{f}\left(1+a^{2}\right) \mathcal{D}_{n}^{q}\left((t-x)^{2}, x\right) \\
& +\omega_{a+1}(f, \delta)\left(1+\frac{1}{\delta} \mathcal{D}_{n}^{q}\left((t-x)^{2}, x\right)\right)^{\frac{1}{2}} .
\end{aligned}
$$

Hence, by using Schwarz inequality and Lemma 2 , for every $q \in(0,1)$ and $x \in[0, a]$

$$
\begin{aligned}
\left|\mathcal{D}_{n}^{q}(f, x)-f(x)\right| \leq & \frac{6 M_{f}\left(1+a^{2}\right) x\left(q[2]_{q}+x\right)}{q[n]_{q}} \\
& +\omega_{a+1}(f, \delta)\left(1+\frac{1}{\delta} \sqrt{\frac{x\left(q[2]_{q}+x\right)}{q[n]_{q}}}\right) \\
\leq & \frac{6 M_{f} a\left(1+a^{2}\right)(2+a)}{q[n]_{q}}+\omega_{a+1}(f, \delta)\left(1+\frac{1}{\delta} \sqrt{\frac{a\left(a+q[2]_{q}\right)}{q[n]_{q}}}\right) .
\end{aligned}
$$

By taking $\delta=\sqrt{\frac{a\left(q[2]_{q}+a\right)}{q[n]_{q}}}$ we get the assertion of our theorem.

\section{Higher order moments and an asymptotic formula}

Lemma 3 ([6]) Let $0<q<1$, we have

$$
\mathcal{B}_{n, q}\left(t^{3}, x\right)=\frac{1}{[n]_{q}} x+\frac{1+2 q}{q^{2}} \frac{[n+1]_{q}}{[n]_{q}^{2}} x^{2}+\frac{1}{q^{3}} \frac{[n+1]_{q}[n+2]_{q}}{[n]_{q}^{2}} x^{3},
$$




$$
\begin{aligned}
\mathcal{B}_{n, q}\left(t^{4}, x\right)= & \frac{1}{[n]_{q}^{3}} x+\frac{1}{q^{3}}\left(1+3 q+3 q^{2}\right) \frac{[n+1]_{q}}{[n]_{q}^{3}} x^{2} \\
& +\frac{1}{q^{5}[2]_{q}}\left(1+3 q+5 q^{2}+3 q^{3}\right) \frac{[n+1]_{q}[n+2]_{q}}{[n]_{q}^{3}} x^{3} \\
& +\frac{1}{q^{6}[2]_{q}[3]_{q}[4]_{q}}\left(1+3 q+5 q^{2}+6 q^{3}+5 q^{4}+3 q^{5}+q^{6}\right) \\
& \times \frac{[n+1]_{q}[n+2]_{q}[n+3]_{q}}{[n]_{q}^{3}} x^{4} .
\end{aligned}
$$

Now, we present higher order moments for the operators (1.4).

Lemma 4 Let $0<q<1$, we have

$$
\begin{aligned}
\mathcal{D}_{n}^{q}\left(t^{3}, x\right)= & \frac{[n+1]_{q}[n+2]_{q}}{q^{3}[n]_{q}^{2}} x^{3}+\left(\frac{1+2 q}{q^{2}} \frac{[n+1]_{q}}{[n]_{q}^{2}}+\frac{(2+q) q}{[n]_{q}}+\frac{(2+q)}{[n]_{q}}\right) x^{2} \\
& +\left(\frac{1}{[n]_{q}^{2}}+\frac{(2+q) q}{[n]_{q}^{2}}+\frac{(1+q) q^{2}}{[n]_{q}^{2}}\right) x, \\
\mathcal{D}_{n}^{q}\left(t^{4}, x\right) & \left(\frac{\left(1+3 q+5 q^{2}+6 q^{3}+5 q^{4}+3 q^{5}+q^{6}\right)}{q^{6}[2]_{q}[3]_{q}[4]_{q}} \frac{[n+1]_{q}[n+2]_{q}[n+3]_{q}}{[n]_{q}^{3}}\right) x^{4} \\
& +\left(\frac{\left(1+3 q+5 q^{2}+3 q^{3}\right)}{q^{5}[2]_{q}} \frac{[n+1]_{q}[n+2]_{q}}{[n]_{q}^{3}}+\frac{q\left(3+2 q+q^{2}\right)}{[n]_{q}^{3}}[n+1]_{q}[n+2]_{q}\right) x^{3} \\
& +\left(\frac{\left(1+3 q+3 q^{2}\right)}{q^{3}} \frac{[n+1]_{q}}{[n]_{q}}+\frac{(1+2 q)\left(3+2 q+q^{2}\right)}{q[n]_{q}^{3}}[n+1]_{q}\right. \\
& \left.+\frac{q^{2}\left(3+4 q+3 q^{2}+q^{3}\right)}{[n]_{q}^{2}}+\frac{q\left(3+4 q+3 q^{2}+q^{3}\right)}{[n]_{q}^{3}}\right) x^{2} \\
& +\left(\frac{1}{[n]_{q}}+\frac{q\left(3+2 q+q^{2}\right)}{[n]_{q}^{3}}\right. \\
& \left.+\frac{q^{2}\left(3+4 q+3 q^{2}+q^{3}\right)}{[n]_{q}^{3}}+\frac{q^{3}\left(1+2 q+2 q^{2}+q^{3}\right)}{[n]_{q}^{3}}\right) x .
\end{aligned}
$$

The proof of Lemma 4 can be obtained by using Lemma 3 .

We consider the following classes of functions:

$$
\begin{aligned}
& C_{m}[0, \infty):=\left\{f \in C[0, \infty): \exists M_{f}>0|f(x)|<M_{f}\left(1+x^{m}\right) \text { and }\|f\|_{m}:=\sup _{x \in[0, \infty)} \frac{|f(x)|}{1+x^{m}}\right\} \\
& C_{m}^{*}[0, \infty):=\left\{f \in C_{m}[0, \infty): \lim _{x \rightarrow \infty} \frac{|f(x)|}{1+x^{m}}<\infty\right\}, \quad m \in \mathbb{N} .
\end{aligned}
$$

Theorem 3 Let $q_{n} \in(0,1)$, then the sequence $\left\{\mathcal{D}_{n}^{q_{n}}(f)\right\}$ converges to $f$ uniformly on $[0, A]$ for each $f \in C_{2}^{*}[0, \infty)$ if and only if $\lim _{n \rightarrow \infty} q_{n}=1$. 
Theorem 4 Assume that $q_{n} \in(0,1), q_{n} \rightarrow 1$ and $q_{n}^{n} \rightarrow$ a as $n \rightarrow \infty$. For any $f \in C_{2}^{*}[0, \infty)$ such that $f^{\prime}, f^{\prime \prime} \in C_{2}^{*}[0, \infty)$ the following equality holds

$$
\lim _{n \rightarrow \infty}[n]_{q_{n}}\left(\mathcal{D}_{n}^{q_{n}}(f ; x)-f(x)\right)=\left(x^{2}+2 x\right) f^{\prime \prime}(x)
$$

uniformly on any $[0, A], A>0$.

Proof Let $f, f^{\prime}, f^{\prime \prime} \in C_{2}^{*}[0, \infty)$ and $x \in[0, \infty)$ be fixed. By using Taylor's formula, we may write

$$
f(t)=f(x)+f^{\prime}(x)(t-x)+\frac{1}{2} f^{\prime \prime}(x)(t-x)^{2}+r(t ; x)(t-x)^{2},
$$

where $r(t ; x)$ is the Peano form of the remainder, $r(\cdot ; x) \in C_{2}^{*}[0, \infty)$ and $\lim _{t \rightarrow x} r(t ; x)=0$. Applying $\mathcal{D}_{n}^{q_{n}}$ to (4.1), we obtain

$$
[n]_{q_{n}}\left(\mathcal{D}_{n}^{q_{n}}(f ; x)-f(x)\right)=\frac{1}{2} f^{\prime \prime}(x)[n]_{q_{n}} \mathcal{D}_{n}^{q_{n}}\left((t-x)^{2} ; x\right)+[n]_{q_{n}} \mathcal{D}_{n}^{q_{n}}\left(r(t ; x)(t-x)^{2} ; x\right) .
$$

By the Cauchy-Schwarz inequality, we have

$$
\mathcal{D}_{n}^{q_{n}}\left(r(t ; x)(t-x)^{2} ; x\right) \leq \sqrt{\mathcal{D}_{n}^{q_{n}}\left(r^{2}(t ; x) ; x\right)} \sqrt{\mathcal{D}_{n}^{q_{n}}\left((t-x)^{4} ; x\right)} .
$$

Observe that $r^{2}(x ; x)=0$ and $r^{2}(\cdot ; x) \in C_{2}^{*}[0, \infty)$. Then it follows from Theorem 3 and Lemma 4, that

$$
\lim _{n \rightarrow \infty} \mathcal{D}_{n}^{q_{n}}\left(r^{2}(t ; x) ; x\right)=r^{2}(x ; x)=0
$$

uniformly with respect to $x \in[0, A]$. Now from (4.2), (4.3) and Remark 2 , we get immediately

$$
\lim _{n \rightarrow \infty}[n]_{q_{n}} \mathcal{D}_{n}^{q_{n}}\left(r(t ; x)(t-x)^{2} ; x\right)=0 .
$$

Then, we get the following

$$
\begin{aligned}
& \lim _{n \rightarrow \infty}[n]_{q_{n}}\left(\mathcal{D}_{n}^{q_{n}}(f ; x)-f(x)\right) \\
& \quad=\lim _{n \rightarrow \infty}\left(\frac{1}{2} f^{\prime \prime}(x)[n]_{q_{n}} \mathcal{D}_{n}^{q_{n}}\left((t-x)^{2} ; x\right)+[n]_{q_{n}} \mathcal{D}_{n}^{q_{n}}\left(r(t ; x)(t-x)^{2} ; x\right)\right) \\
& \quad=\left(x^{2}+2 x\right) f^{\prime \prime}(x) .
\end{aligned}
$$

\section{Competing interests}

The authors declare that they have no competing interests.

\section{Authors' contributions}

All authors contributed in preparing the manuscript equally.

\section{Author details}

'School of Applied Sciences, Netaji Subhas Institute of Technology, Sector 3 Dwarka, New Delhi 110078, India. ${ }^{2}$ Department of Mathematics, Kwangwoon University, Seoul 139-701, S. Korea. ${ }^{3}$ Division of General

Education-Mathematics, Kwangwoon University, Seoul 139-701, S. Korea. 


\section{Acknowledgements}

The work was done while the first author visited Division of General Education-mathematics, Kwangwoon University, Seoul, South Korea for collaborative research during June 15-25, 2010.

Received: 7 May 2012 Accepted: 4 June 2012 Published: 22 June 2012

\section{References}

1. Agrawal, PN, Mohammad, AJ: Linear combination of a new sequence of linear positive operators. Rev. Unión Mat. Argent. 44(1), 33-41 (2003)

2. Aral, A, Gupta, V: Generalized q-Baskakov operators. Math. Slovaca 61(4), 619-634 (2011)

3. Aral, A, Gupta, V: On the Durrmeyer type modification of the $q$-Baskakov type operators. Nonlinear Anal. 72(3-4), 1171-1180 (2010)

4. DeVore, RA, Lorentz, GG: Constructive Approximation. Springer, Berlin (1993)

5. Ernst, T: The history of q-calculus and a new method. U.U.D.M Report 2000, 16, ISSN 1101-3591, Department of Mathematics, Upsala University (2000)

6. Finta, Z, Gupta, V: Approximation properties of q-Baskakov operators. Cent. Eur. J. Math. 8(1), 199-211 (2010)

7. Gupta, V, Radu, C: Statistical approximation properties of q-Baskakov-Kantorovich operators. Cent. Eur. J. Math. 7(4), 809-818 (2009)

8. Kac, VG, Cheung, P: Quantum Calculus. Universitext. Springer, New York (2002)

9. Kim, T, Rim, S-H: A note on the q-integral and q-series. Adv. Stud. Contemp. Math. (Pusan) 2, $37-45$ (2000)

10. Kim, T: $q$-Bernoulli numbers and polynomials associated with Gaussian binomial coefficients. Russ. J. Math. Phys. 15, 51-57 (2008)

11. Kim, T: q-Bernoulli numbers associated with q-Stirling numbers. Adv. Differ. Equ. 2008, Art. ID 743295 (2008)

12. Koornwinder, TH: q-special functions, a tutorial. In: Gerstenhaber, M, Stasheff, J (eds.) Deformation Theory and Quantum Groups with Applications to Mathematical Physics. Contemp. Math., vol. 134. Am. Math. Soc., Providence (1992)

13. Phillips, GM: Bernstein polynomials based on the q-integers. Ann. Numer. Math. 4, 511-518 (1997)

14. Wang, X: The iterative approximation of a new sequence of linear positive operators. J. Jishou Univ. Nat. Sci. Ed. 26(2), $72-78$ (2005)

doi:10.1186/1029-242X-2012-144

Cite this article as: Gupta et al.: q-analogue of a new sequence of linear positive operators. Journal of Inequalities and Applications 2012 2012:144.

\section{Submit your manuscript to a SpringerOpen ${ }^{\circ}$ journal and benefit from:}

- Convenient online submission

- Rigorous peer review

- Immediate publication on acceptance

- Open access: articles freely available online

- High visibility within the field

- Retaining the copyright to your article 